\title{
Osteomyelitis of the ribs in Ibadan
}

\author{
O OSINOWO, OA ADEBO, AO OKUBANJO
}

From the Departments of Surgery and Radiology, University College Hospital, Ibadan, Nigeria

ABSTRACT Sixteen patients with pyogenic osteomyelitis of the ribs are reported; ages ranged from 3 months to 42 years and 10 were female. Right sided ribs were affected in 10 cases and single ribs in 12 . Antecedent causes included empyema thoracis (56\%) and blunt chest trauma (19\%). Fourteen out of 16 patients presented with discharging chest wall sinuses and five patients had associated swellings on the chest wall. The duration of symptoms ranged from two to 36 months. The most common microorganism isolated was Staphylococcus aureus (50\%). Rib excision (with drainage of the empyema in two cases) was curative in all 16 cases in this study.

Osteomyelitis of the ribs appears to be a rare disease in both the tropical and the temperate areas of the world. ${ }^{1-3}$ Adeyokunnu and Hendrickse, ${ }^{4}$ in a review of 63 cases of salmonella osteomyelitis seen in Nigerian children, found no case of rib osteomyelitis. Similarly, Dich et al $^{1}$ reviewed 163 cases of osteomyelitis in infants and children over 15 years and found only one case $(0.6 \%)$ affecting the ribs.

Osteomyelitis of the ribs has been found in association with childhood empyema, ${ }^{5-7}$ Staphylococcus aureus infection, ${ }^{1}$ sickle cell anaemia, and infection due to Salmonella and Klebsiella species and Proteus rettgeri. ${ }^{3}$ Osteomyelitis arising in ribs adjacent to sites of intubation in the chest in children with empyema has been documented by Osinowo et al. ${ }^{7}$

The present report reviews 16 cases of rib osteomyelitis treated by our cardiothoracic unit over seven and a half years. From this experience and a review of the published reports we consider the aetiology, pathogenesis, clinical presentation, and results of treatment of the disease.

\section{Methods}

The records of all patients with rib osteomyelitis admitted to the unit from January 1975 to May 1983 formed the basis of this prospective study.

The data extracted from each patient's record included the age, sex, clinical presentation, radiological findings, mode and results of treatment, and the results of bacteriological and routine hae-

Address for reprint requests: Dr O Osinowo, Department of Surgery, University College Hospital, Ibadan, Nigeria.

Accepted 31 May 1985 motological investigations and haemoglobin electrophoresis.

Rib resection was carried out in all the patients under general anaesthesia.

\section{Results}

The clinical features of the 16 patients are shown in the table. The ages of the patients ranged from 3 months to 42 years (mean 9.32 (SD 11.38) years). Ten were female and six male (M:F = 1:1.7). All six patients in whom the haemoglobin genotype was known were AA. The packed cell volume ranged from $26 \%$ to $52 \%$ (mean $34.7(7.0), \mathrm{n}=10)$ and the white blood cell count from 3700 to $1450010^{9} / 1$ (mean 9006 (4051), $\mathrm{n}=9$ ).

Ribs on the right side were affected in 10 cases and on the left side in six. A single rib was affected in 12 cases $(75 \%)$ while two ribs were affected simultaneously in three cases $(19 \%)$. In the remaining case three ribs were affected. Empyema thoracis and blunt chest trauma were the commonest causes of the osteomyelitis. Five of the 16 patients $(31 \%)$ presented with chest wall swellings. Discharging sinuses were seen in 14 of the patients $(87.5 \%)$ and were multiple in three. Two patients $(12.5 \%)$ had no sinuses on presentation. The duration of symptoms was known in 12 patients and ranged from 2 to 36 months (mean 7.8 (SD 8.9)).

The most common microorganism isolated was Staphylococcus aureus, which was present in half the cases. In one case each Pseudomonas aeruginosa and Klebsiella sp were isolated in addition to $S$ aurens. Radiologically bone destruction and patchy necrosis were seen in all the affected ribs. Three patients presented with expansion of the affected rib (fig 1) 


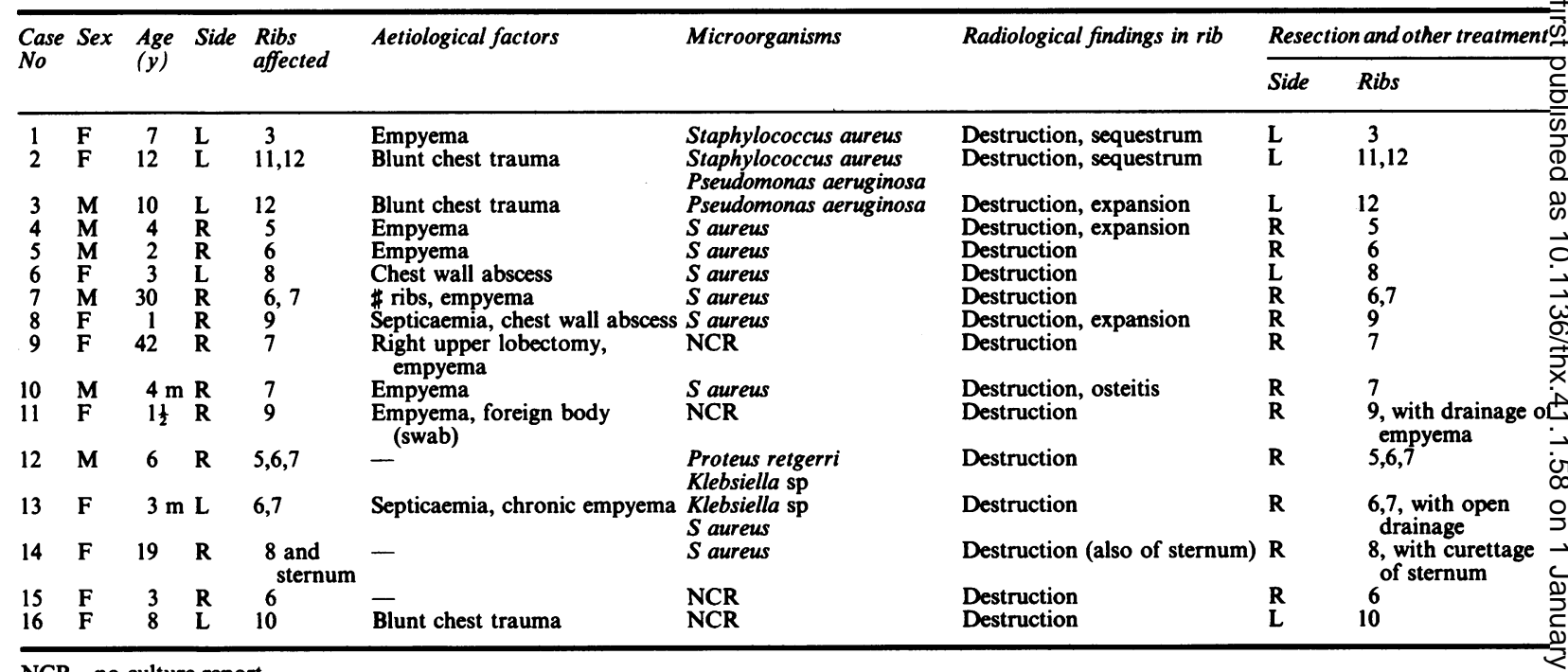

NCR-no culture report.

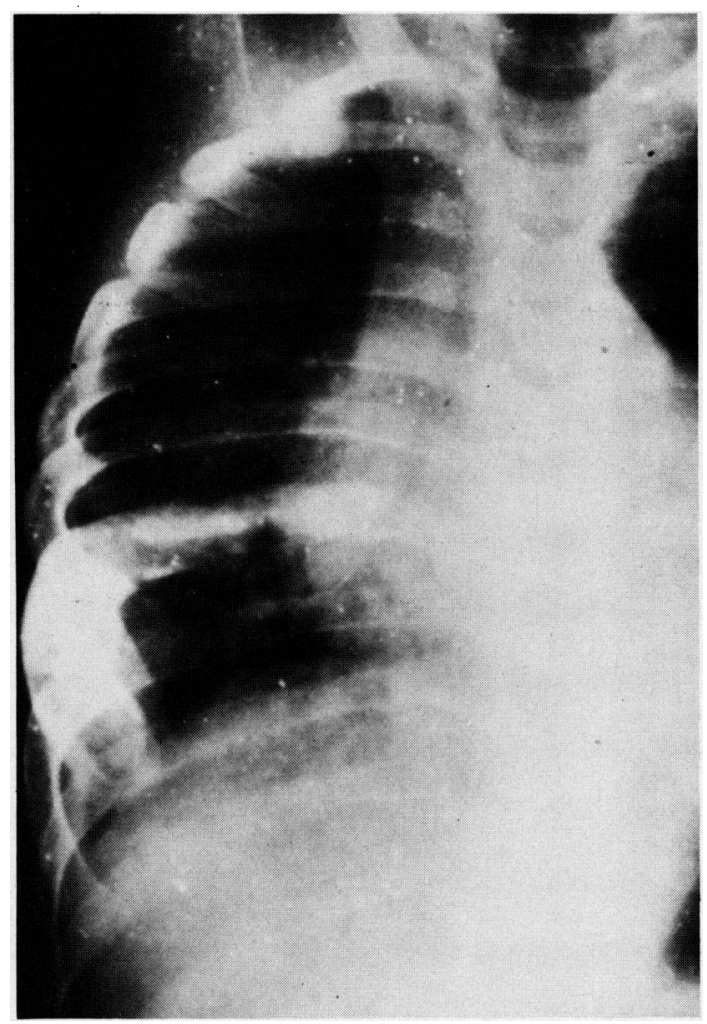

Fig 1 Radiograph showing osteomyelitis of the right 7 th rib in 4 month old boy after chest tube drainage for empyema.

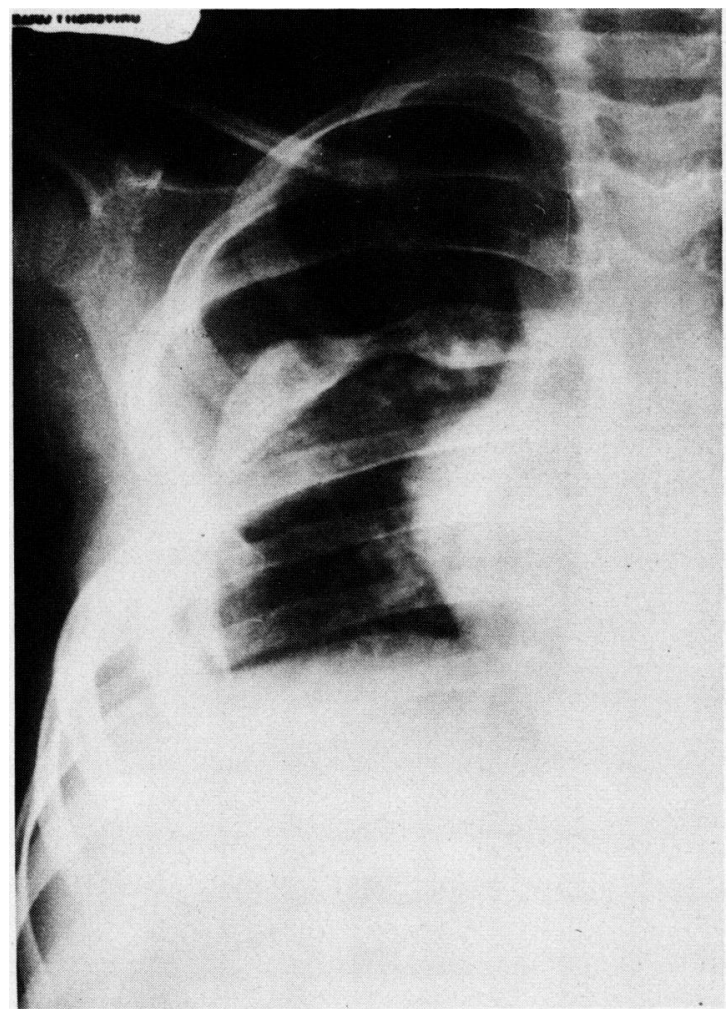

Fig 2 Radiograph of 4 year old boy with discharging chest wall sinus and osteomyelitis destruction of the right 5th rib. There is a sequestrum in the anterior portion of the affected rib. 
while in two cases sequestra were visible radiologically (fig 2).

All the patients responded well to rib resection, combined with drainage of the empyema in two cases.

\section{Discussion}

Osteomyelitis of the ribs appears to be more common in children than in adults and in our series was related mainly to empyema thoracis and blunt chest trauma. It may also occur at the site of chest intubation, as a result of pressure necrosis cased by large or stiff chest tubes. $^{7}$ In a study of 178 children with empyema Osinowo $\mathrm{et} \mathrm{al}^{7}$ reported a $1.1 \%$ incidence of rib osteomyelitis. Antecedent blunt chest trauma may cause fracture of the rib and compromise its blood supply, which in the presence of subsequent infection may lead to the development of osteomyelitis. The importance of septicaemia and haemoglobinopathies as causative factors in rib osteomyelitis has been emphasised ${ }^{4}$ but these are rarely encountered.

Patients with osteomyelitis of the rib commonly present with a chronic discharging sinus ${ }^{13}$ and sometimes swelling ${ }^{3}$ in the chest wall, as has been confirmed in our study.

We cannot readily explain the frequency of rib osteomyelitis on the right side in this study (10/16), but disease of the right 6 th and 7 th ribs may be due to the frequent use of the 6th intercostal space for chest intubation for empyema. Although Komolafe $^{3}$ reported one case of bilateral rib osteomyelitis out of the five they studied, we encountered no such caseseven the cases in which more than one rib was affected were all ipsilateral.

The diagnosis of rib ostoeomyelitis is readily confirmed radiologically by the presence of expansion and destruction of the rib, patchy necrosis, and extra- pleural swelling of soft tissue. Occasionally a sequestrum may be present.

Despite reports that salmonella species are the major causative organisms of osteomyelitis in those with haemoglobinopathies, ${ }^{4}$ Staphylococcus aureus remains the commonest cause of osteomyelitis in general. ${ }^{1-3}$

Resection of the rib offers the best treatment for pyogenic rib osteomyelitis, ${ }^{38}$ as the complete cure of all of our 16 patients has shown.

We thank the biomedical communications unit of the College of Medicine, University of Ibadan, for preparing the figures and Mrs TT Odebunmi for typing the manuscript.

\section{References}

1 Dich VQ, Nelson JD, Haltalin KC, et al. Osteomyelitis in infants and children. A review of 163 cases. Am J Dis Child 1975;129:1273-8.

2 Mollan RAB, Piggot J. Acute osteomyelitis in children. J Bone Joint Surg 1977;59:2-7.

3 Komolafe F. Pyogenic osteomyelitis of the rib in children. Pediatr Radiol 1982;12:245-8.

4 Adeyokunnu AA, Hendrickse RG. Salmonella osteomyelitis in childhood. A report of 63 cases seen in Nigerian children of whom 57 had sickle cell anaemia. Arch Dis Child 1980;55:175-84.

5 Aderele WI, Stevenson CE, Antia AU. Empyema in childhood: a review of 116 cases. Nigerian Journal of Paediatrics 1974;1:20-5.

6 Adebonojo SA, Osinowo O, Oyemade OA. Surgical management of empyema in children. Nigerian Medical Journal 1970:469-74.

7 Osinowo O, Adebonojo SA, Adebo OA, et al. Childhood empyema in Ibadan, Nigeria. Nigerian Medical Journal 1982;12:337-47.

8 Guest JL, Anderson JN. Osteomyelitis involving adjacent ribs. JAMA 1978;239:133. 